\title{
Augmentation of the Oswestry Parawalker Orthosis by means of Surface Electrical Stimulation: Gait Analysis of Three Patients
}

\author{
M. McClelland, B.Sc., F.R.C.S., B. J. Andrews, Ph.D., J. H. Patrick, \\ F.R.C.S., P. A. Freeman, F.R.C.S. and W. S. El Masri, F.R.C.S. \\ Orthotic Research $\mathcal{E}$ Locomotor Assessment Unit, Robert fones $\mathcal{E}$ Agnes Hunt \\ Orthopaedic Hospital, Oswestry, Shropshire, England; The Bioengineering Unit, \\ University of Strathclyde, Glasgow, Scotland and The West of Scotland Spinal \\ Injuries Unit, Philipshill Hospital, Glasgow, Scotland
}

\section{Summary}

The Oswestry 'Parawalker' orthosis has been supplied to 15 adult thoracic level complete paraplegic patients enabling them to achieve a reciprocal gait with an inherent low energy demand. In order to further off-load the work demands on the upper limb girdle musculature during ambulation, three of these patients have undergone electrical stimulation of the gluteal muscles in stance phase. This was shown to increase the stability of the adduction and also provided forward propulsion by driving the stance-leg into extension. Bilateral stimulation of the quadriceps muscles has been shown to facilitate standing and sitting in the orthosis.

Using a Kistler force platform, the crutch impulse can be seen to be reduced by as much as $36^{\circ}{ }_{0}$. We speculate that this leads to a similar order of reduction in the work done by the upper limbs in these patients.

Key words: Paraplegia; Hybrid orthosis; Functional electrical stimulation.

\section{Introduction}

In patients having preserved lower motorneurone pathways, there is the possibility of using functional electrical stimulation in conjunction with mechanical bracing to improve the overall orthotic solution. These devices are referred to as a Hybrid Orthosis, a concept initially put forward by Tomovic et al. in 1972. Prototype hybrid orthoses for paraplegics have been reported (Schwirtlich \& Popovic, 1984, Andrews \& Bajd, 1984, Patrick, 1985).

The 'Parawalker' orthosis, which permits adult paraplegics to ambulate reciprocally, has developed out of the hip guidance orthosis designed by Rose (1979) for spina bifida children. It was recognised at an early stage that the adults did not perform as well as the children. Clinical observation of the differences suggested two main reasons for this. The first is the inconvenience and physical effort required of an adult to stand and sit in the orthosis. The second was due to the deformation of the orthosis at the hip joint, on the stance side, in the heavier adult. Children are remarkably adept at independently donning and standing up in the Parawalker. In adults the process is more laborious often 
requiring the assistance of others or the use of mechanical lifting devices (Griffiths et al., 1980). Electrical stimulation has been applied bilaterally to the quadriceps muscles to assist in standing and sitting manoeuvres in the Parawalker (Andrews \& Bajd 1984) in a manner similar to that initially described by Bajd et al., 1981. Initial trials with electrical stimulation of the hip abductors on the stance side during ambulation have shown that this technique can be used to resist the deformation of the orthosis (Patrick \& McClelland, 1985). Judicious placement of the electrodes can also produce hip extension and thus help drive the swing leg forward (Butler \& Major, 1984).

This paper describes the application of a 2-channel electrical stimulator for assisting adult paraplegics to stand and walk in the Parawalker. Preliminary results are presented of gait laboratory experiments carried out with paraplegic patients using gluteal stimulation during ambulation.

\section{Methods}

In order to facilitate standing and sitting in any location a wheelchair mounted support was developed (Andrews et al., 1986). This detachable support shown in Figure 1 comprises a hinged armrest fitted with a telescopic section that can extend to the floor. When not in use, the support folds away into the space occupied by a conventional armrest. Standing in the Parawalker was performed by bilateral stimulation of the quadriceps muscles. These muscles were initially conditioned by an exercise programme comprising daily application of cyclical electrical stimulation (Kralj et al., 1983). The standing sequence is illustrated in Figure 1. The subject positions himself forward on the wheelchair cushion with his body weight over his feet. The Parawalker knee joints are fitted with spring loaded bail locks that are disengaged at this stage. He then presses the $S T A N D$ switch and assumes the position as shown. After a few seconds delay, to allow him to prepare for standing, the maximal stimulus is applied. Throughout the manoeuvre the subject regulates the amount of body weight taken by his upper limbs so as to produce a smooth movement. Once upright the knee joints lock out automatically and the subject switches off the stimulus to avoid fatiguing the quadriceps. With practice the movement can be performed smoothly with a minimum of upper limb effort. The subject then engages the hip joint motion restraints and dons the elbow crutches in preparation for walking.

Sitting back down in the wheelchair is performed in a reversed sequence. The subject backs into position within the support frame and disengages the hip joint motion restraints. Maximal quadriceps stimulation is applied and the knee joint locks disengaged using cable pulls. The $S I T$ switch is pressed with a few seconds delay to allow the subject time to grip the supports before the quadriceps stimulus slowly diminishes. The subject guides his progressive descent into the wheelchair using his upper limbs. As an alternative to using cable pulls the practiced subject may choose to lean backwards against the front edge of the wheelchair cushion thereby depressing the posterior bail lock releasing bars as the quadriceps stimulus begins to diminish.

A pair of Cooper elbow crutches were modified to incorporate push-buttons into the handlegrips so that the 2-channel stimulator could be operated by the 


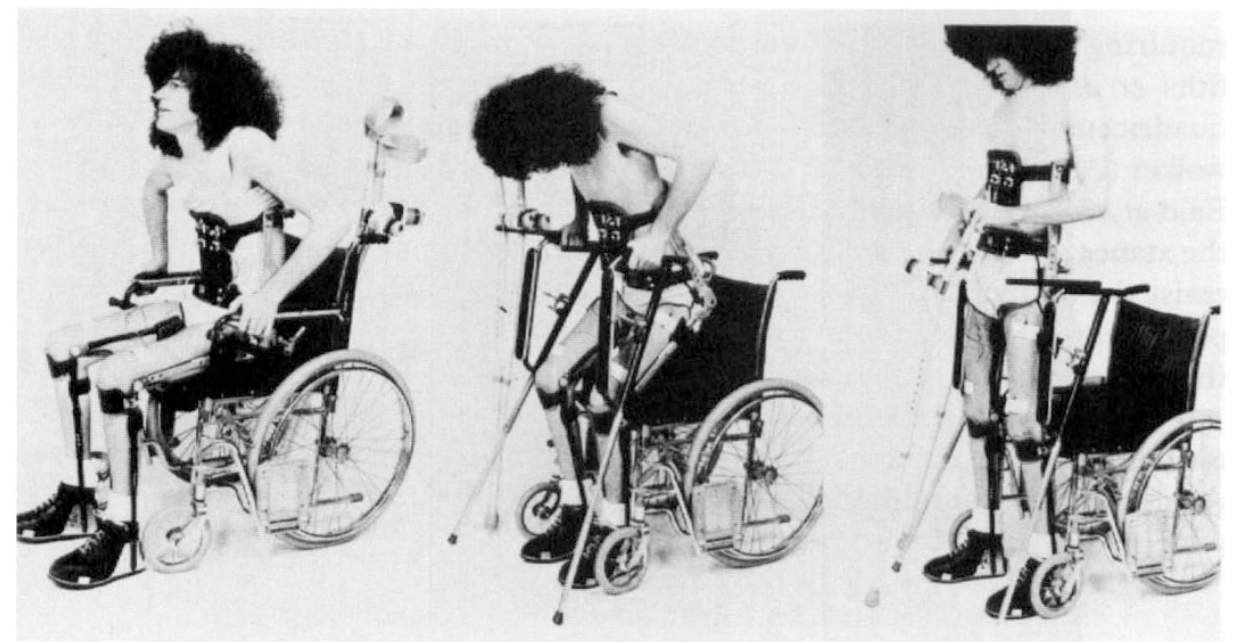

Figure 1 Paraplegic subject (C.F. age 26 years, mass $74 \mathrm{~kg}$, height $1.9 \mathrm{~m}$, lesion T 4 complete, 8 years post injury) demonstrating the use of the wheelchair support and quadriceps stimulation to assist standing in the 'Parawalker' orthosis.

patient's index fingers. Leads were passed through the hollow structure of the crutches and emerged just below the elbow clips and connected with the stimulator. The stimulator was set up in such a way that pressing a crutch button resulted in stimulation of the contralateral hip abductors. This meant that, in training, the patient had only to remember to press the button as he pushed himself over to gain leg clearance. This proved remarkably easy to learn.

Three mid-thoracic level paraplegics (T4, T6 and T7 complete) who were all experienced 'Parawalker' users, underwent a 2 months course of gluteal stimulation to condition these muscles (Kralj et al., 1973). Following this course, trials were undertaken in which the patients negotiated the 20 foot gait laboratory walkpath, first without and then with gluteal stimulation. The patients were asked to ambulate in such a way that a single crutch strike could be recorded from a Kistler force platform imbedded in the floor. They were asked to walk with a steady rhythm and, if possible, not to look at the force plate. If it was felt that the patient had altered his stride in order to purposely strike the force plate then the subsequent reading was not accepted. The data from the force platform was captured using a Commodore 4032 microcomputer and could be output in graphical form as shown in Figure 2 or absolute values given for the three orthogonal components of the reaction force and its duration of action. The speed of ambulation, without and with stimulation was also recorded.

In this application, the quadriceps and gluteal muscles are stimulated during different locomotor activities. Only two channels of stimulus are required provided there is a convenient means to connect the stimulator outputs to the two respective sets of electrodes. For this purpose a two channel stimulator was developed at the Bioengineering Unit. The stimulator is shown in Figure 1 and provides monophasic, rectangular pulses with a repetition rate of 20 per second with an effective pulse duration of $300 \mu \mathrm{s}$. The stimulator can deliver a maximum 

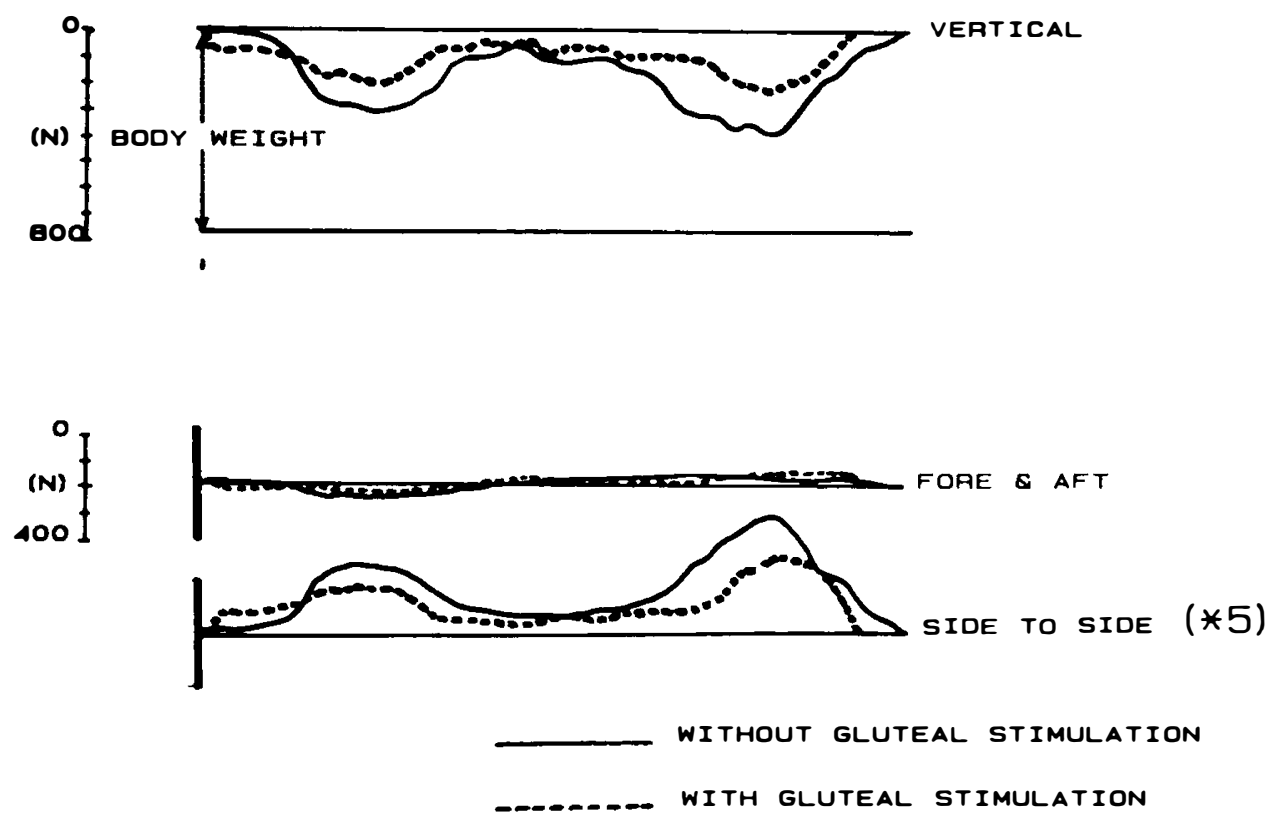

Figure 2 Graphs of the vertical, side to side and fore and aft components of the crutch/ground reaction with and without functional stimulation of the gluteal muscles on the stance side.

of $120 \mathrm{ma}$ into a $1 \mathrm{kohm}$ load. A slide switch is used to connect the output to the relevant electrodes. Control of quadriceps stimulus is provided by a panel mounted $S T A N D / S I T$ switch. For convenience, self adhesive electrodes were used (Myocare 3M Ltd.).

\section{Preliminary results}

All three patients commented that the addition of gluteal stimulation made ambulation easier with less effort being needed to bring the swing leg forward. Figure 2 shows the typical graphical output for the 3 components of crutch forces against time. The effect of adding gluteal stimulation (dotted line) can be seen both as a reduction in the force size and its duration of action. The decrease in floor contact time is a direct consequence of their increased speed of ambulation, Figure 3. The product of force and its duration of application is given by the area under the curve and is called the force/time integral (or 'impulse').

The crutch/ground reaction will have an identical reaction at the shoulder and will, therefore, relate to the work done by the shoulder girdle musculature in producing this reaction. Hence, reduction of the work done by the shoulder girdle will be reflected in reduced crutch impulse.

Figure 4 shows the total crutch impulse (the vector sum of the three components), both with and without stimulation, for the three patients in the trial. The addition of gluteal stimulation brought about reductions in crutch impulse of $36 \%, 19 \%$ and $28 \%$ respectively. It is of interest to note that patient A.E., who had the greatest reduction, had been in the programme longest, followed by patient D.L. who had the $28 \%$ reduction. 


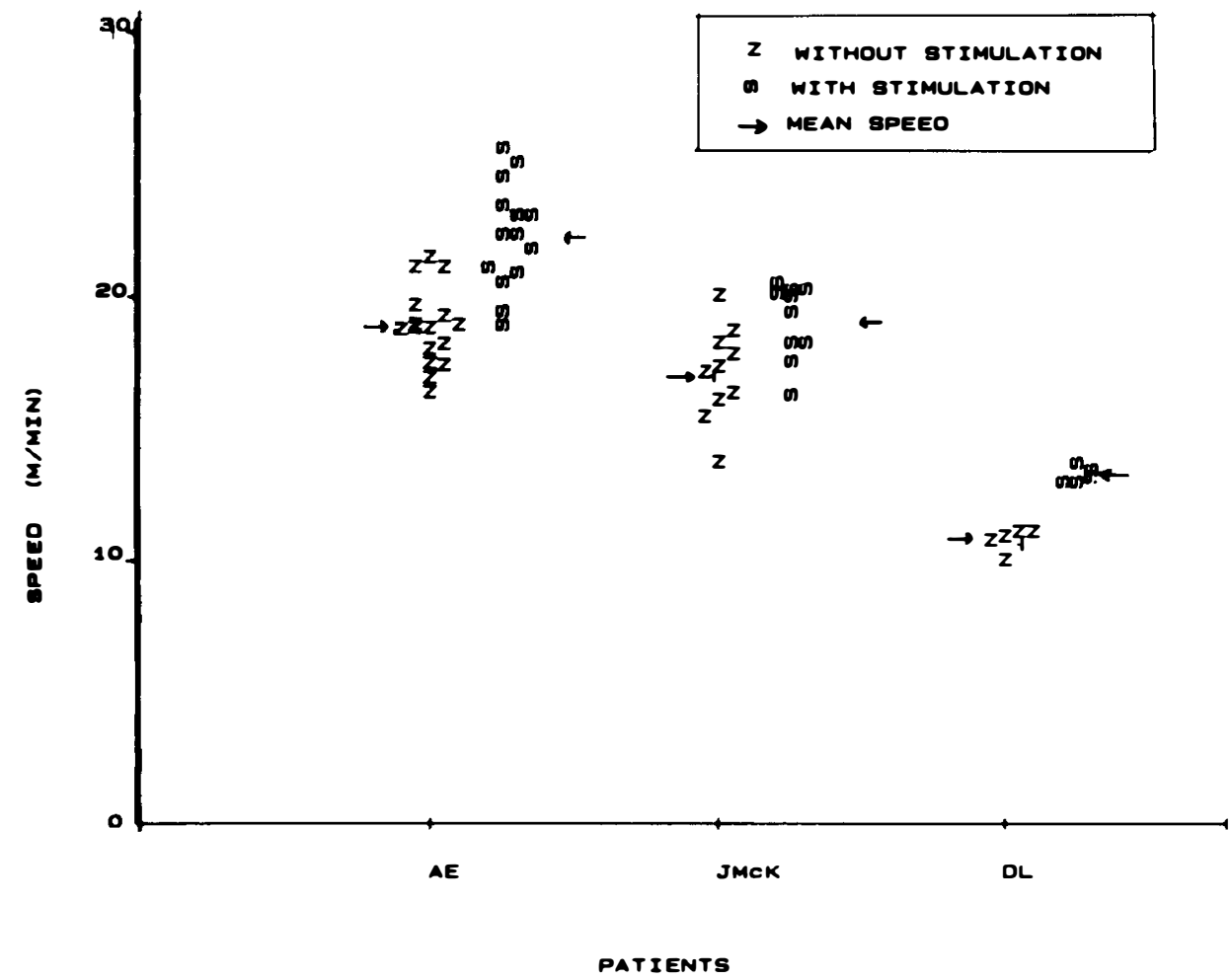

Figure 3 Speed of ambulation for the three patients with and without functional stimulation.

\section{Discussion}

The use of quadriceps stimulation was found to greatly assist standing and sitting. The patients quickly learned the technique and commented on the ease with which they were able to stand and sit.

The success of the 'Parawalker' as a low-energy reciprocating orthosis lies in the maintenance of its rigidity. Because the knees and ankles are fixed by the device and the hips are limited to one degree of freedom within a limited arc, it is possible for the patient to make use of the transfer of momentum between body segments and thus reduce energy costs.

During single leg stance the body centre of mass produces an adducting moment about the stance hip. This moment, although resisted by the crutch on the opposite side, creates an outward bowing of the orthosis at its most flexible region - the hip joint. If uncorrected, this adduction of the legs prevents proper swing-leg clearance. Further tilting towards the stance side is therefore required to gain clearance and this results in still more bending of the orthosis. A greater effort must be applied through the crutches to prevent the line of action of the centre of mass from passing lateral to the support foot.

It is well recognised that shoulder girdle muscle fatigue is a major limiting factor in paraplegic ambulation (Gordon and Vanderwalde, 1956). The 'Parawalker' orthosis goes some way to reducing the effort required from these 


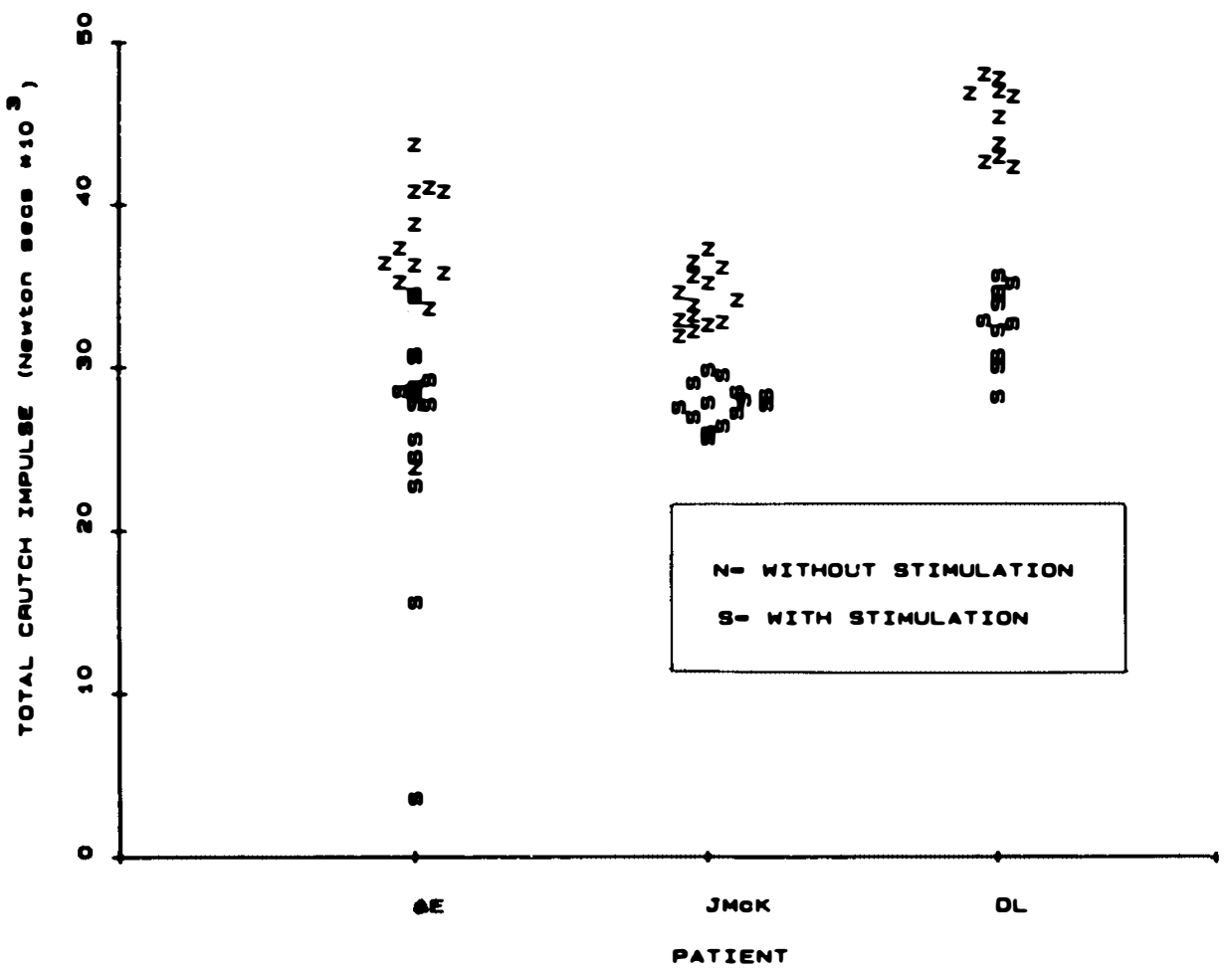

Figure 4 Total crutch impulse for the three patients with and without stimulation.

muscles. That further reduction is achieved by gluteal stimulation in the first half of stance phase is evidenced by the reduced crutch impulse. Also, as any gluteal muscle group is required to contract for less than half of the full stride time, the stimulation induced fatigue of three muscles is minimised (Benton et al., 1981).

\section{Conclusions}

The use of quadriceps stimulation was subjectively found to be useful in assisting patients to stand and sit in the 'Parawalker' orthosis.

The addition of functional gluteal stimulation to patients ambulating in the 'Parawalker' orthosis results in improvement in performance which can be measured objectively as a significant increase in walking speed. More importantly, there is a decrease in the work done by the shoulder girdle muscles as is evidenced by the reduction in crutch/ground impulse.

\section{Acknowledgement}

The authors acknowledge the financial support of the Department of Health and Social Security and the Scottish Home and Health Department. The clinical work was conducted at the Orthotic Research and Locomotor Assessment Unit in Oswestry and the West of Scotland Spinal Injury Unit at the Philipshill Hospital in Glasgow. 


\section{References}

ANDREwS BJ, BAJD T 1984 Hybrid Orthoses for Paraplegics. Proc. Supl. 8th Internat. Symp. on External Control of Human Extremities, Dubrovnik. Sept. 1984, Y.C.E.A. Belgrade.

Andrews BJ, Baxendale RH, Katakis J, Cliquet A, Freeman PA, Paul JP. Restoration of Locomotion in Spinal Cord Injured Subjects using F.E.S., Digest No. 1986/6 I.E.E. Colloquim on F.E.S. Jan. 1986. 1-7.

Bajd T, KRALJ A, SEGa J, TURK R (1981) Use of a 2 channel Functional Electrical Stimulation to stand paraplegic patients. Physical Therapy 61:526.

Benton LA, Baker LL, Bowman BA, WATERS RL 1981 Functional Electrical Stimulation - A Practical Clinical Guide. 2nd Edition, Rancho Los Amigos Rehab. Eng. Centre, Downey, California.

Butler PB, Major RE, Patrick JH (1984) The technique of using hip guidance orthosis with crutches. Prosthetics \& Orthotics International 8:33-38.

Gordon EE, Vanderwalde H 1956 Energy Requirements in Paraplegic Ambulation. Arch. Phys. Med. \& Rehabili., 276-285.

Griffiths JC, Henshaw JT, Haywood OB, Taylor AG (1980) Clinical Applications of the Paraplegic Swivel Walker. Journal of Biomedical Engineering 2:250-256.

Kralj A, Grobelnik S 1979 Functional Electrical Stimulation. A new hope for paraplegic patients? Bulletin of Prosthetic Research, 75-101.

Patrick JH, MCClelland MR 1985 Low Energy Cost Reciprocal Walker for the Adult Paraplegic. Paraplegia, 1985.

Rose GK 1979 The Principles and Practice of Hip Guidance Articulations. Prosthetics \& Orthotics International 3:37-43.

Schwirtlich L, Popovic D 1984 Hybrid Orthoses for Deficient Locomotion. Proc. Supl. 8th Internat. Symp. on External Control of Human Extremities, Dubrovnik, Sept. 1984, Y.C.E.A. Belgrade.

Tomovic R, Vukobratovic M, Vodovnik L 1972 Hybrid Actuators for Orthotic Systems: Hybrid Assistive Systems. Proc. 4th Internat. Symp. on External Control of Human Extremities, Dubrovnik, Aug./Sept. 1972, Y.C.E.A. Belgrade. 